\title{
Zur Kenntnis der Ferrocyanide.
}

\author{
Von \\ J. Messner.
}

(Mitteilung aus dem technologischen Institut der Universität Würzburg.)

Die vielen Versuche, mehr Licht in die Konstitution des sog. Berliner und Turnbulls Blau zu bringen, welche die Litteratur aufweist und die geringen Resultate dieser Arbeiten beweisen schon die Sehwierigkeit solcher Untersuchungen.

Meine mehrjährigen Arbeiten über Ferrocyanide haben nur einige neue Verbindungen zu Tage befördert, die ich unter dem Titel „Krystallisierte Kupferferrocyanide“ in dieser Zeitschrift ${ }^{1}$ beschrieben habe. Letztere solien heute mit zum Abschlufs gebracht werden.

Die Veröffentlichung nachstehender Arbeit über amorphe Ferrocyanide hat lediglich den $Z$ weck, Fachgenossen, die sich mit diesen und ähnlichen Körpern beschäftigen oder beschäftigen werden, einige Anhaltspunkte zu geben, die ihnen möglicherweise von Nutzen sein können, die ihnen auch die Anstellung des einen oder des anderen Versuches ersparen.

Die Hauptschwierigkeit, die Konstitution des Turnbulls Blau und des Berliner Grün zu bestimmen, liegt darin, dafs diese Körper durch zersetzende Reagentien in andere Komponenten gespalten werden, als man erwarten sollte. So wird z. B. Turnbulls Blau, das der Darstellung nach Ferroferricyanid sein sollte und vielleicht auch ist, durch Alkalien nicht in Ferrohydroxyd und Ferricyanalkali, sondern in Ferrihydroxyd und Ferrocyanalkali verwandelt, gerade wie dies beim Berliner Blau der Fall ist. Dieser Umstand allein ist es wohl, dals die meisten Chemiker das Turnbulls Blau für identisch halten mit Berliner Blau, und um diese Ansicht theoretisch zu rechtfertigen, nimmt man an, dals beim Versetzen von Ferricyankali mit Ferrosalzen die letzteren durch die oxydierende Wirkung des $\mathrm{K}_{3} \mathrm{FeCy}_{6}$ in Ferrisalze verwandelt würden, wobei sich das $\mathrm{K}_{3} \mathrm{FeCy}_{6}$ in $\mathrm{K}_{4} \mathrm{FeCy}_{6}$ reduziert. Diese beiden Umsetzungsprodukte sollen dann in normaler Weise Berliner Blau bilden und es wäre

\footnotetext{
1 Diese Zeitschr. 8, 368.
} 
somit die Existenz eines Ferroferricyanids in Frage gestellt. Allein so gut mir die oxydierende Wirkung des $\mathrm{K}_{3} \mathrm{FeCy}_{6}$ bekannt ist, eluenso ist mir die reduzierende Eigenschaft des $\mathrm{K}_{4} \mathrm{FeCy}_{6}$ bekannt. So werden durch $\mathrm{K}_{3} \mathrm{FeCy}_{6}$ Bleisalze in alkalischer Lösung zu Bleisuperoxyd oxydiert, es wird aber auch das Wilusamsonsche Violett, das sog. Ferrikaliumferrocyanid durch $\mathrm{K}_{4} \mathrm{FeCy}_{6}$ in Ferrokaliumferrocyanid umgesetzt, wobei $\mathrm{K}_{4} \mathrm{FeCy}_{6}$ in $\mathrm{K}_{3} \mathrm{FeCy}_{6}$ oxydiert wird. Man sieht also schon aus diesem einen Beispiele, dals ebensogut beim Mischen von $\mathrm{K}_{4} \mathrm{FeCy}_{6}$ und $\mathrm{FeCl}_{3}$ zuerst $\mathrm{K}_{3} \mathrm{FeCy}_{6}$ und $\mathrm{FeCl}_{2}$ entstehen könnte, welche sich zu Ferroferricyanid zusammenlagern. Es lassen sich auch noch andere Hypothesen über den Verlauf des genannten Vorganges aufstellen und thatsächlich sind solche auch aufgestellt worden, allein die für diese Hypothesen in der litteratur angeführten Beweise stehen alle auf so schwachen Füfsen, dafs man ihnen absolut kein Vertrauen schenken kann. Je länger man mit diesen Körpern arbeitet, desto mehr kommt man zu der Überzeugung, dals man über den Vorgang bei der Bildung des Berliner und Turnbulls Blau wohl leicht eine Behauptung aufstellen kann, den Beweis aber schuldig bleiben mufs. Die Beweisführung ist dadurch unmöglich, dafs die zur Darstellung genannter blauer Körper verwendeten Stoffe sich momentan umsetzen und so etwaige Zwischenprodukte nicht gefalst werden können.

Der Umstand, dals sowohl Berliner Blau als auch Turnbulls Blau durch Alkalien immer in Ferrihydroxyd und Ferrocyanalkali zerlegt wird, kann zu einer Beweisführung niemals benutzt werden, was schon daraus hervorgeht, dafs Ferrohydroxyd und Ferricyanalkali sich in Ferrihydroxyd und Ferrocyankali umsetzen. Es muls also aus Turnbulls Blau durch Alkalien auch in dem Falle, dals es Ferroferricyanid ist, Ferrihydroxyd und Ferrocyanalkali entstehen. Wie bedeutungslos die Alkalizersetzung für die Konstitutionsfrage ist, geht auch aus den folgenden Betrachtungen des Berliner Grüns und des WiLlisamsonschen Violetts hervor.

Ich versuchte daher das Blau, das ich auf die verschiedensten Arten darstellte, durch Borax, durch primäres, sekundäres und tertiäres Natriumphosphat, durch Oxyde von Leicht- und Schwermetallen wie $\mathrm{MgO}, \mathrm{ZnO}, \mathrm{HgO}$, CdO etc. zu zersetzen, allein ohne jeden $\mathrm{Er}$ folg, da die hierbei entstehenden Zersetzungsprodukte einen Schluls auf die Konstitution des Blaus nicht zulassen. Ebensowenig kommt man zum Ziele, wenn man das Blau durch Kochen mit konz. Lösungen der Schwermetalle zu zerlegen sucht. Es entsteht bei dieser Ope- 
ration immer mehr Eisenoxydul als sich mit der Theorie verträgt, weil hei diesen Zersetzungen das frei werdende Cyan reduzierend wirkt.

Ich destillierte das Blau mit konz. Schwefelsäure. Dabei entsteht schweflige Säure und konnte infolgedessen eine quantitative Bestimmung des in Lösung gegangenen Rückstandes nicht beweisliefernd sein. Deshalb stellte ich denselben Versuch mit $50 \%$ Schwefelsäure an unter Ersatz des abdestillierenden Wassers. Es fanden sich nach vollständiger Zersetzung des zugesetzten Blaus Eisenoxydul und Eisenoxyd in Mengenverhältnissen vor, die mit der Theorie nicht im Einklang standen, obwohl die Destillation in einer Wasserstoffatmosphäre vorgenommen worden war. Dabei lieferten mehrere Versuche, mit gleichen Substanzmengen ausgeführt, stets wechselnde Resultate. Denselben Erfolg zeigte eine Zersetzung der blauen Körper mit konz. Phosphorsäure.

Durch konz. Salzsäure wird sowohl Berliner als auch Turnbulls Blau grölstenteils zerstört. Wenn man das Blau mit konz. Salzsäure in der Kälte behandelt, so geht ein Teil davon in Lösung. Diese gelbe Lösung durch Asbest filtriert, scheidet beim Verdünnen mit Wasser wieder Blau ab, wobei aber immer überschüssiges Eisen in Lösung bleibt, da durch die Salzsäure ein 'Teil des Cyans ausgetrieben wurde. Einige Berliner Blau, die ich auf diese Art zersetzte, zeigten viel in Lösung gegangenes Eisenoxyd neben wenig Oxydul, während die untersuchten Turnbulls Blau viel Eisenoxydul neben wenig Oxyd lieferten. Einen Schlufs daraus zu ziehen scheint mir zu gewagt und zwar aus folgendem Grund: Es ist sehr leicht möglich, dafs las von mir dargestellte Turnbulls Blau weniger kompakt als das Berliner Blau war und infolgedessen von der Salzsäure heftiger angegriffen wurde. Hierbei wird mehr Cyan aus der Verbindung gelöst und dieses wirkt dann durch seine gröfsere Quantität auch stärker reduzierend, also dals mehr Eisenoxydul in Lösung geht. Wenn diese Ansicht auch nicht bewiesen werden kann, so ist immer die Mrïglichkeit eines solchen Vorganges vorhanden und sind eventuelle Täuschungen nicht ausgeschlossen. Ich unterliels also unter so zweifelhaften Verhältnissen eine quantitative Untersuchung.

Man sollte nun allerdings glauben, dafs die quantitative Analyse den besten Aufschlufs geben mülste, denn die theoretischen Formeln $\mathrm{Fe}_{4}\left(\mathrm{FeCy}_{6}\right)_{3}$ für Berliner Blau und $\mathrm{Fe}_{3}\left(\mathrm{FeCy}_{6}\right)_{2}$ für Turnbulls Blau zeigen doch quantitative Verschiedenheiten von Eisen und Cyan. 
Allein diese beiden Körper sind nicht so rein zu erhalten, als dals man wie bei krystallisierten Verbindungen auf genau zur Theorie stimmende Analysenresultate hoffen dürfte, was auch untenstehende Analysenresultate beweisen, und zweitens ist trotz der scheinbar grolsen Verschiedenheit der Formeln die quantitative Zusammensetzung fast gleich. Die theoretisch berechneten Prozentzahlen sind

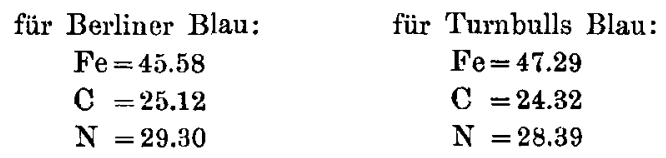

Wenn also irgend ein Berliner oder Turnbulls Blau ein mit der Theorie genau übereinstimmendes Resultat liefert, so ist das mehr der zufälligen Zusammensetzung als etwa einer reinen chemischen Individualität zuzuschreiben.

Ich stelle in folgendem einige Darstellungsweisen und Analysenresultate zusammen, beschränke mich aber, um Raum zu sparen, nur auf kurze Angaben und auf die aus den Analysen berechneten Prozentzahlen.

1. Eine Lösung von $3 \mathrm{~g} \mathrm{~K}_{4} \mathrm{FeCy}_{6}$ wurde in eine Lösung von $6 \mathrm{~g} \mathrm{FeCl}_{3}$ filtriert und der Niederschlag mit Wasser sorgfältig durch Dekantieren ausgewaschen. Das Blau wurde im Vakuum über Schwcfelsäure getrocknet.

2. Reine Ferrocyanwasserstoffsäure wurde durch überschüssiges $\mathrm{FeCl}_{3}$ gefällt und das so entstandene Blau ausgewaschen und gotrockuet wie bei 1.

3. $3.5 \mathrm{~g}$ Ferrosulfat in Wasser golöst, mit $5 \mathrm{~g}$ Ferrocyankali gefällt und mit Salpetersäure oxydiert, lieferten ein Blau, das wie oben gewaschen und getrocknet wurde.

4. Berliner Grün (aus $\mathrm{K}_{3} \mathrm{FeCy}_{6}$ und Chlor dargestellt; siehe später!) wurde durch Erhitzen auf $150^{\circ} \mathrm{C}$. in Blau übergeführt, zuerst mit verdünnter Salzsäure, dann mit Wasser gewaschen etc.

5. Ferricyankali wurde mit verdünnter Sehwefelsäure so lange gekocht, bis der dabei entstandene Niederschlag völlig blau geworden war. Das erhaltene Blau wurde mit Wasser bis zur Entfernung der Schwefelsäure gewaschen und getrocknet.

6. Berliner Grün (aus Wruframsoss Violett durch Oxydation mit Salpetersäure dargestellt) wurde, wie bei 4. beschrieben, durch Erhitzen in Blau üborgefuihrt.

Die hier angeführten blauen Körper lieferten bei der Analyse folgende Prozentzahlen:

\begin{tabular}{|c|c|c|c|c|c|c|}
\hline $\begin{array}{c}1 \\
F_{e}=45.88\end{array}$ & $\begin{array}{c}2 . \\
45.72\end{array}$ & $\begin{array}{c}3 . \\
38.49\end{array}$ & $\begin{array}{c}4 . \\
45.29\end{array}$ & $\begin{array}{c}5 . \\
46.68\end{array}$ & $\begin{array}{c}6 . \\
45.11\end{array}$ & $\begin{array}{l}\text { Theorie: } \\
\mathrm{Fe}_{4}\left(\mathrm{FeCy}_{\mathrm{\theta}}\right)_{3} \\
\quad 45.58\end{array}$ \\
\hline$C=24.11$ & 25.28 & 23.72 & 25.28 & 24.23 & 25.43 & 25.12 \\
\hline$=28.39$ & 28.97 & 27.66 & 29.56 & 28.15 & 29.66 & 29.30 \\
\hline$K=1.61$ & - & 11.01 & - & 0.91 & 一 & - \\
\hline
\end{tabular}


Diese Werte sind die Mittelzahlen aus je drei Analysen.

Wenn man diese Zahlen betrachtet, so findet man, dals 2, 4 und 6 am besten zur Theorie passen, weniger 1 und noch weniger 3, dagegen stimmt 5 viel besser auf die theoretischen Zahlen des Turnbulls Blau.

7. $8.8 \mathrm{~g}$ Ferrosulfat wurde durch $5 \mathrm{~g}$ Ferricyankali gefält und das entstandene Blau mit Wasser gut ausgewaschen und im Vakuum getrocknet.

8. Reine Ferricyanwasserstoffsäure wurde durch Ferrosulfat gefällt ete.

$\begin{array}{ccc}7 . & 8 . & \text { Theorie: } \mathrm{Fe}_{3}\left(\mathrm{FeCy}_{6}\right)_{2} \\ \mathrm{Fe}=45.93 & 46.07 & 47.29 \\ \mathrm{C}=23.57 & 24.88 & 24.32 \\ \mathrm{~N}=27.49 & 29.03 & 28.39 \\ \mathrm{~K}=2.93 & - & -\end{array}$

Diese Resultate der beiden Turnbulls Blau stimmen besser auf die theoretischen Zahlen des Berliner Blau. Man sieht also, dals durch die quantitative Analyse ein Schlufs auf die Konstitution der blauen Körper nicht gemacht werden kann.

Es ist nicht meine Aufgabe, die Angaben der Litteratur zu kritisieren, aber an dieser Stelle möchte ich den, der bei etwaigen Arbeiten oder einer sonstigen Information halber die Nachschlagebücher oder die Originalarbeiten zu Rate zieht, darauf aufmerksam machen, dal's gerade in den Angaben über Ferrocyanide eine Unsicherheit herrscht, die es sehr wünschenswert erscheinen liefse, wenn sich jemand finden würde, der sich der allerdings mühsamen und zeitraubenden Arbeit unterzöge, das Chaos zu sichten. Es darf also niemand wundern, wenn er nach den Angaben der Litteratur arbeitend nicht genau dieselben Resultate findet, wie sie in der Litteratur angegeben sind. Nicht immer sind die Autoren schuld an den Irrtümern, die zuweilen schon in die Lehrbücher übergegangen sind; es liegt meistens an den Versuchen, die nicht in der nötigen Zahl ausgeführt wurden und an der zu geringen Zahl der ausgeführten quantitativen Analysen, wenn solche Litteraturangaben mit den Thatsachen wenig oder gar nicht übereinstimmen. Die Eigenschaften der auf verschiedene Arten dargestellten blauen Eisencyanide sind nicht immer dieselben und weichen, was Farbe, Kupferglanz, Löslichkeit und Zersetzlichkeit bei Einwirkung von Reagentien anbetrifft, voneinander soweit $a b$, dafs man nicht immer im stande ist, ein allgemeines Gesetz aufzustellen oder mit Bestimmtheit die Eigenschaften des zu bereitenden blauen Körpers vorauszusagen. Den Grund für solche Erscheinungen zu suchen, hat meiner Ansicht nach keinen Wert, so lange in dieser Angelegenheit 
viel wichtigere Fragen zu lösen sind. Um ein Beispiel hier anzuführen, will ich auf die Löslichkeit des Berliner Blaus hinweisen. Es ist eine allbekannte Thatsache, dafs Berliner Blau in wasserlöslicher Form erhalten werden kann (siehe Daмmer III, $374 \mathrm{u.} \mathrm{377),}$ aber nicht immer erhält man auf die dort angegebene Art lösliches Berliner Blau und meistens ist das, was man für eine Lösung hält, nur ein sehr fein verteilter Niederschlag, der sich in einem Zeitraum von Tagen oder Wochen absetzt. Man findet oft, dafs die blauen Niederschläge bei langem Auswaschen scheinbar in Lösung gehen, aber alle diese Lösungen setzen $a b$, wenn man sie genügend lange stehen läfst. Ebenso unrichtig ist es, $\mathrm{zu}$ behaupten, dals Berliner Blau in Oxalsäure löslich sei, denn diese Behauptung trifft nicht immer zu, darf also auch nur bedingt ausgesprochen werden. Von 20 auf verschiedene Arten dargestellten Blau lösten sich vier nur scheinbar in Oxalsäure, indem nach ein- bis zweitägigem Stehen sich alles Blau aus der wasserhellen Oxalsäurelösung abgeschieden hatte, während andere nach monatelangem Stehen aus der Oxalsäurelösung sich nicht abschieden. Von dem aus Blausäurerückstand durch Oxydation erhaltenen Blau ist die Unlöslichkeit in Oxalsäure schon längst bekannt. Auf letzteres komme ich übrigens noch besonders zurlick.

Wie leicht man sich übrigens auch nach vielen Versuchen täuschen kann, mag folgender Versuch, den ich vor zwei Jahren mehr als zwanzigmal anstellte, und der immer dasselbe Resultat lieferte, illustrieren. Bei der Darstellung von Blausäurerückstand verwendete ich Ferrocyankali und Schwefelsäure in Mengenverhältnissen, dafs Ferrocyankali in geringem Überschufs vorhanden war und so bei der Destillation nicht vollständig zur Zersetzung gelangte. Dabei fand ich die merkwürdige Thatsache, dafs das in Lösung restierende Ferrocyankali durch Ferrisalze in eine purpurrote Lösung verwandelt wurde, durch Ferrosalze farblos blieb und beim Stehen an der Luft dieselbe rote Färbung annahm. Durch langes Stehenlassen, schneller nach kurzem Erhitzen, erhielt ich aus dieser Lösung einen flockigen rotvioletten Niederschlag, der sich nach dem Trocknen mit derselben roten Farbe in Oxalsäure löste, sich aber längstens nach zweitägigem Stehen wieder aus der Lösung abschied. Ich konnte diesen Körper leider nicht rein erhalten, da er sich wegen seiner ungeheuren Feinheit auf dem Filter nicht sammeln liefs und beim Auswaschen mit Wasser durch Dekantieren in Lösung ging, bevor alies Kaliumsulfat aus der Mutterlauge entfernt war. Wichtigere 
Arbeiten veranlalsten mich damals, die Sache einstweilen auf sich beruhen zu lassen, bis ich vor kurzer Zeit behufs eines Vortrages diesen Körper zur Demonstration anfertigen wollte und zu meinem Erstaunen fand, dals ich ihn absolut nicht mehr erhalten kounte; wie ich die Versuchsbedingungen auch einrichtete. Was daran schuld ist, kann ich mir vorderhand nicht erklären, ich vermute jedoch, dafs ich zu den ersten Versuchen ein Ferrocyankali verwendet hatte, das durch irgend eine geringe Verunreinigung $\mathrm{zu}$ genanntem roten Körper Veranlassung gab. Es wäre mir sehr interessant, zu wissen, ob diese eigentümliche Erscheinung auch schon anderweitig angetroffen wurde oder nicht.

\section{Berliner Grün.}

Die Untersuchung des Berliner Grüns hatte mehr Erfolg. Es giebt wobl kaum einen Körper, für den so viele Formeln aufgestellt wurden, als für diesen. So hat Williamson' ${ }^{2}$ die Formel $\mathrm{Fe}_{5}(\mathrm{CN})_{7}$, Pelouze ${ }^{2} \mathrm{Fe}_{3}(\mathrm{CN})_{4}$, Roscoe und Schorlemurer ${ }^{3} \mathrm{Fe}_{9}(\mathrm{CN})_{24}$, Kenule 4 $\mathrm{Fe}_{13}(\mathrm{CN})_{36}$ und ERLENMEYER ${ }^{5} \mathrm{Fe}_{11}(\mathrm{CN})_{36}$ angegeben und man ist im allgemeinen der Ansicht gewesen, dal's Berliner Grün ein Körper sei, der eine Art Doppelsalz sei von Ferriferrocyanid und Ferriferricyanid. ERLenmeyer hält es für ein Ferroferrisalz der Ferricyanwasserstoffsäure. Diese Ansichten stützen sich zumeist auf die von Williamson und Pelouze gelieferten Resultate und ferner auf die Zersetzung des Berliner Grüns durch Alkalien, wobei Ferround Ferricyanalkali neben Ferrihydroxyd entstehen.

Eine unglücklichere Reaktion als letztere hätte man aber in der That nicht finden können. Ich habe bei meinen Versuchen die Erfahrung gemacht, dafs bei der Zersetzung von Ferricyaniden durch Alkalien immer eine Neigung des Ferricyanrestes zu erkennen ist, in den Ferrocyanrest überzugehen, was vielleicht auf die reduzierende Wirkung von intermediär gebildetem Cyanalkali zurückzuführen ist. So erhält man z. B. aus Ferricyankupfer und Alkali bei der Zersetzung sowohl Ferri- als auch Ferrocyanalkali; ja noch auffallender ist die Thatsache, dals Ferricyankupfer in Wasser aufgeschlämmt im Laufe einiger Monate fast vollständig in Ferrocyankupfer sich

'Williamson, Lieb. Ann. 57, 232.

2 Pesouze, Ann. Chim. Phys. 62, 69.

3 Roscoe und Schorlemmer (1889), II. 542.

4 Kekulé, Lehrb. d. org. Chem. 1, 332.

s Erlenmeyer, siehe Dammer (1893), III. 378. 
umsetzt. Bei der Zersetzung von Ferricyanblei mit Natriumkarbonat entsteht ebenfalls nebenbei Ferrocyannatrium; beim Kochen von Ferricyankali mit Kaliumbydroxyd bildet sich Ferrocyankali, und so liefsen sich noch mehr Beispiele anführen, welche beweisen, dafs die Zersetzungsprodukte der Ferricyanide durch Alkalien einen Schluls auf ihre Konstitution nicht zulassen.

Schon Wriliamson, der durch Oxydation seines nach ilum benannten Violetts einen grünen Körper erhielt, vermutete, dals dieser grüne Körper identisch sei mit dem von Pelovze aus Ferricyankali und Chlor dargestellten Grün. In der That ist es auch so. Denn man erhält durch Oxydation aller Eisencyanide Berliner Grün, welches das höchste Oxydationsprodulst zu sein scheint. Sogar Berliner Blau lälst sich durch mäfsig konz. Salpetersäure in Grün überführen. In nachstehendem sollen einige Entstehungsarten des Berliner Grüns angegeben und die entsprechenden Analysenresultate kurz angeführt werden.

Die folgenden Präparate wurden nach ihrer Darstellung alle mit Salzsäure ausgekocht und im Vakuum über Schwefelsäure getrocknet.

1. In eine $5 \%$ ige Lösung von Ferricyankali wurde Chlor bis zur Sättigung eingeleitet und dann die Lösung zum Sieden erhitzt. Das so entstehende Grün ist von heller Furbe und geht beim Erhitzen auf $150^{\circ} \mathrm{C}$. in ein sehr schönes violettblaues Berliner Blau über. Letzteres zeichnet sich von dem gewöhnliehen Berliner Blau besonders dadurch aus, dafs es keinen Kupferglanz besitzt. In seiner Farbennuance stimmt es fast vollkommen mit dem Wrichamsonschen Violett überein. Dieses wie die folgenden Berliner Grün verglimmen schon bei $200^{\circ} \mathrm{C}$.

2. $3 \mathrm{~g}$ Ferricyankali, in $60 \mathrm{~g}$ Wasser gelöst, wurden mit $5,5 \mathrm{~g}$ Salpetersäure (spez. Gew. 1,4) gekocht. Das so entstandene Grün ist dunkler als das vorige und liefert beim Erhitzen auf $150^{\circ} \mathrm{C}$. ein entsprechend dunkleres Berliner Blau.

3. Blausäurerückstand wurde durch Kochen mit 5\% iger Salpetersäure in Grün verwandelt. Es stellt ein dunkelgrünes Pulver dar.

4. Blausäurerïckstand in Wasser aufgeschlämmt wurde durch Chlor oxydiert und so in Grün verwandelt.

5. Ferricyankali wurde mit $10 \%$ iger Schwefelsäure gekocht und sofort nach Bildung eines grünen Niederschlages in kaltes Wasser gegossen. Letzteres ist deshalb nötig, da sich das so gebildete Grün sehr leicht in Berliner Blan verwandelt.

6. Es wurde eine möglichst konz. Lösung von sogenanntem Ferriferricyanid dargestellt durch Versetzung von Ferrisulfat mit Bleiferricyanid. Diese bekannte braune Lösung setzt bei längerem Stehen einen grünen Niederschlag ab. Auf diese Art erhält man meistens ein sehr schönes helles Grün. 
7. Ein aus Ferrosulfat und Ferrocyankali erhaltener Niederschlag wurde mit Salpetersäure oxydiert, bis er grün geworden war.

8. Eine Mischung von 2,4 $\mathrm{g}$ Berliner Blau und $3,1 \mathrm{~g}$ Blausäurerückstand, die ich zu einem anderen Versuche dargestellt hatte, wurde mit $10 \%$ iger Salpetersäure oxydiert.

9. Ferricyankali und Ferrisalze geben in konz. Lösung nach kurzein Stehen einen grünen Niederschlag.

Die so dargestellten Grün lieferten folgende Analysenresultate:

\begin{tabular}{|c|c|c|c|c|c|c|c|c|c|}
\hline & 1 & 2. & 3. & 4. & 5. & 6. & 7. & 8. & 9. \\
\hline $\mathrm{Fe}$ & $=36.17$ & 29.05 & 37.67 & 31.97 & 37.04 & 34.32 & 37.15 & 37.82 & 29.27 \\
\hline Xy & $=46.75$ & $37 .: 5$ & 49.89 & 42.21 & 48.32 & 44.98 & 48.08 & 47.90 & 37.84 \\
\hline $\mathrm{H}_{9} \mathrm{O}$ & $=17.00$ & 31.09 & 11.39 & 23.32 & 13.42 & 20.51 & 13.83 & 12.87 & 31.60 \\
\hline & $=1.53$ & 1.58 & 1.57 & 164 & 0.46 & - & 0.59 & 0.73 & 0.98 \\
\hline
\end{tabular}

Die hier angegebenen Prozentzahlen sind immer das Mittel aus drei Analysen. Die Analyse selbst wurde wie folgt ausgeführt: Der Kohlenstoff wurde durch Verbrennung in Kupferoxyd bestimmt und daraus das Cyan berechnet. Der Wassergehalt wurde bei der Verbrennung ermittelt. Das Eisen und Kalium, welch letzteres nur als Verunreinigung betrachtet werden kann, wurde nach Abrauchen der Substanz mit Schwefelsäure auf die gewöhnliche Art als Eisenoxyd und Kaliumsulfat zur Wägung gebracht. Das Trocknen im Vakuum setzte ich so lange fort, bis ein anscheinend lufttrockenes Präparat vorlag, das zum Wägen geeignet war. Ein Trocknen bis zur Gewichtskonstanz unterliels ich, da ich dadurch einer zu weit gehenden Zersetzung vorbeugen wollte. Rechnet man die aus der Analyse erhaltenen Zahlen auf kalifreie und wasserfreie Substanz um, so dafs man die Verhältniszahlen von Eisen und Cyan bilden kann, erhält man folgende Prozentzahlen:

$\begin{array}{ccccccccc}1 . & 2 . & 3 . & 4 . & 5 . & 6 . & 7 . & 8 . & 9 . \\ \mathrm{Fe}=43.60 & 43.17 & \mathbf{4 3 . 0 2} & 43.04 & 43.00 & \mathbf{4 3 . 2 8} & 43.64 & \mathbf{4 3 . 7 7} & \mathbf{4 3 . 4 1} \\ \mathrm{Cy}=56.36 & 56.31 & 56.97 & 56.96 & 56.12 & 56.72 & 56.48 & 55.88 & 56.27\end{array}$

Berechnet man die von Pelodze und Wildiamson gefundenen Prozentzahlen ebenso auf wasserfreie Substanz, so ergeben sich folgende Zahlen:

$\begin{array}{lc}\text { Pelotze } & \text { Williamson } \\ \mathrm{Fe}=44.68 & 43.00 \\ \mathrm{Cy}=\mathbf{5 5 . 3 1} & \mathbf{5 8 . 4 6}\end{array}$

Daraus stellte Pelouze die Formel $\mathrm{Fe}_{3} \mathrm{Cy}_{8}$ und Wiluiamson die Formel $\mathrm{Fe}_{5} \mathrm{Cy}_{14}$ auf. 
Allein wie bedeutungslos sowohl diese beiden Formeln als die von Roscoe, Kerule und ErLenmeyer angegebenen Formeln sind, sieht man sofort aus folgender Betrachtung: Berechnet man z. B. aus den oben unter Nr. 1 angegebenen Prozentzahlen eine Formel, so erbält man auf 1 Eisen 2.77 Cyan, also annähernd der Formel $\mathrm{FeCy}_{3}$. Dieses Verhältnis der Atomzahlen wird natürlich nicht gestört, wenn man dasselbe mit 2, 3, 4 etc. multipliziert, d. h. man erhält dann die Verhältniszahlen:

$\begin{array}{llr}1: 2.77 & 5: 13.85 & 9: 24.93 \\ 2: 5.54 & 6: 16.62 & 11: 30.47 \\ 3: 8.31 & 7: 19.39 & 13: 36.01 \\ 4: 11.08 & 8: 22.16 & \end{array}$

Ganz ähnliche Zahlen berechnen sich auch aus den übrigen oben angegebenen Analysenresultaten. Aus diesen Atomverhältniszahlen sieht man sofort, dal's die oben genannten Autoren mit ihren Formeln einer so gut Recht hat als der andere. Denn
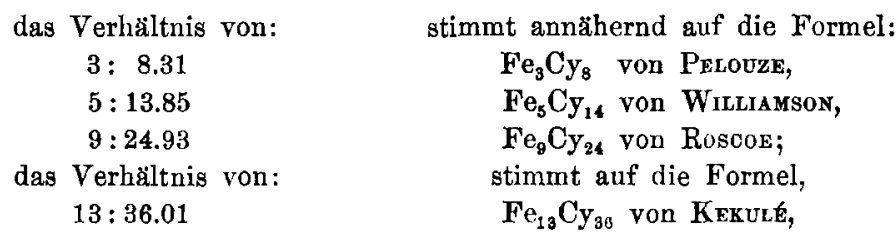

nur das Verhältnis $11: 30.47$ stimmt mit der Formel $\mathrm{Fe}_{11} \mathrm{Cy}_{36}$ von ERLENMEYer gar nicht. Bei der Wahl, aus den 11 angegebenen Verhältniszahlen die für eine Formel richtigste zu finden, dürfte schwierig sein, und mehr als eine Formel lälst sich eben doch nicht aufstellen.

Dieses Mifsverhältnis aufzuklären, schien mir anfangs schwer, allein der Umstand, dafs Berliner Grün sich nicht weiter oxydieren läfst, ohne zu zerfallen, ferner dafs sich Berliner Grün aus dem sogenannten Ferriferricyanid bildete, und das sich aus den Analysen berechnende Verhältnis von Eisen und Cyan, das immer annähernd $1: 3$ ist, weisen direkt darauf hin, dafs Berliner Grün nichts anderes ist, als Ferriferricyanid. Der Beweis hierfür lälst sich nur durch besser stimmende Analysenresultate erbringen, als die oben von mir gefundenen und die von Pelouze angegebenen. Das Resultat WiLLIAMSONS stimmt mit meiner Ansicht fast vollkommen überein, denn $\mathrm{Fe}_{5} \mathrm{Cy}_{14}$ und $\mathrm{Fe}_{5} \mathrm{Cy}_{15}$ (oder $\mathrm{FeCy}_{3}=\mathrm{Fe}_{2} \mathrm{Cy}_{6}=\mathrm{Fr}^{\mathrm{Ir}} \mathrm{eFeCy}{ }_{6}$ ) kommen sich 
sehr nahe, und wenn mall die von WibLIAmson gefundenen, oben angegebenen Zahlen durch die betreffenden Atomgewichte dividiert, so erhält man die Verhältniszahlen $76.8: 224.8$ oder $1: 2.93$.

Was ist nun schuld, dals man bei noch so genauer und vorsichtiger Darstellungsweise des Berliner Grüns keine besser auf die Formel $\mathrm{FeFeCy}_{6}$ stimmenden Analysenresultate gefunden hat? Da man die Eigenschaften des Berliner Grüns kennt, ist es eigentlich sehr zu verwundern, dafs nicht schon längst die Ursache der schlechten Resultate gefunden wurde.

Beim Erwärmen, ja sogar bei gewöhnlicher Temperatur, giebt Berliner Grün Cyan ab und geht teilweise in Berliner Blau über. Dieser Gehalt an Berliner Blau drückt den Cyangehalt mehr oder weniger herunter, ohne dafs man an der grünen Farbe des Präparats eine Veränderung wahrnehmen könnte. Pelouze wufiste, dafs nach seiner Darstellungsweise nebenbei Berliner Blau entstand und suchte letzteres durch Kochen mit konz. Salzsäure zu entfernen, da Berliner Grün gegen Salzsäure ziemlich unempfindlich ist, während Berliner Blau fast vollkommen zerstört wird. Allein beim Auswaschen und Trocknen bildet sich wieder Berliner Blau, wenn man nicht sehr subtil arbeitet. Aber nicht nur beim Trocknen allein, sondern auch unter Einwirkung des Lichtes bildet sich Berliner Blau.

Um also einen Beweis für die quantitative Zusammensetzung des Berliner Grüns liefern zu können, muls das Trocknen vermieden werden und die Einwirkung des Lichtes ausgeschlossen werden. Ich modifizierte daher die von PELouze angegebene Darstellungsweise: An einem vor Tageslicht geschützten Orte leitete ich Chlor in eine Lösung von Ferricyankali bis zur Sättigung. Die Lösung erhitzte ich unter fortwährendem Einleiten von Chlor zum Sieden und liels dann erkalten, ebenfalls unter Chloreinleiten. Auf diese Art ist eine Reduktion des gebildeten Grüns in Blau ausgeschlossen. PELOUZE kochte das Chlor vollkommen weg, wobei sich das Grün zum grofsen Teil durch die kochende Flüssigkeit zersetzte. Das so erhaltene Grün wusch ich bei Lichtabschlufs bis zur völligen Entfernung des Chlors mit kaltem Wasser aus. Von der in Wasser aufgeschlämmten Substanz nahm ich eine ungewogene Menge zur Analyse, die ich nach der von Fresevios angegebenen Methode ausführte. Die Substanz wurde mit Silbernitratlösung bei gelinder Wärme zersetzt, wobei Cyansilber entsteht und Eisen in Lösung geht. Das erhaltene Cyansilber wurde in einem Porzellantiegel bis zur Gewichtskonstanz geglüht und aus dem so erhaltenen Silber 
das Cyan berechnet. Das in Lösung gegangene Eisen wurde nach Entfernung des überschüssigen Silbernitrats mit Ammoniak als Hydroxyd gefällt und als Oxyd zur Wägung gebracht. Ich gebe hier die Resultate dreier Analysen kurz an:

$\left.\begin{array}{cccc}1 . & 2 . & 3 . & \\ \mathrm{Fe}=42.51 & \mathbf{4 1 . 9 1} & 42.31 & \mathbf{4 1 . 7 9} \\ \mathrm{Cy}=57.48 & 58.05 & 57.69 & 58.21\end{array}\right\} \begin{gathered}\text { theoretisch berechnet anf } \\ \text { die Formel FeFeCy. }\end{gathered}$

Diese Zahlen stimmen mit der Theorie so gut überein, als man von einer amorphen Substanz bei der Schwierigkeit ihrer Reindarstellung verlangen kann, und der quantitativen Zusammensetzung nach wäre der Beweis geliefert, dafs Berliner Grün Ferriferricyanid ist.

Man hat bis jetzt jenen rotbraunen Körper, der beim Mischen von Ferrisalzen und Ferricyankali entsteht, für Ferriferricyanid gehalten. Nach den von mir gefundenen Resultaten ist diese Möglichkeit auch nicht ausgeschlossen. Ein Beweis wird sich hierfür allerdings nicht erbringen lassen, solange man diesen braunen Körper nicht rein darstellen kann. Möglicherweise ist der braune Körper die lösliche Modifikation des grünen, die beim Stehen in die unlösliche Modifikation, das Grün, übergeht.

Vollständig unhaltbar ist jedenfalls die Ansicht, wie sie von KeKulÉ und ErLenMerer ausgesprochen wurde. ${ }^{1}$ KeKules Formel $3 \mathrm{FeCy}_{2} .5 \mathrm{Fe}_{2} \mathrm{Cy}_{6}$ stimmt allerdings auf die Zahlen, welche die Analyse des unreinen Berliner Grüns liefert, wie aus obigem ersichtlich, während ERLENMEYens Formel $\mathrm{Fe}_{3} \mathrm{Fe}_{2}\left(\mathrm{FeCy}_{6}\right)_{6}$ bedeutend mehr Cyan beanspruchen würde, als thatsächlich in der Verbindung enthalten ist, aber ganz abgesehen von den quantitativen Verhältnissen läfst sich diese Ansicht mit der Theorie nicht vereinbaren. Wenn man bedenkt, dafs Ferricyankali ein anerkanntes Ferrisalz ist, so ist es nicht möglich zu erklären, wie dieses Ferrisalz durch Chlor, ein so heftiges Oxydationsmittel, teilweise reduziert werden soll. Bei Anwesenheit von überschüssigem Chlor dem in der Verbindung enthaltenen Cyan diese reduzierende Wirkung zuzuschreiben, geht doch kaum an, da man ja sonst Ferrocyankali ebenfalls nicht vollständig in Ferricyankali durch Chlor umwandeln könnte.

Ein Grund zu der Annahme, dals die auf verschiedenem Wege dargestellten Berliner Grün verschiedene Körper seien, ist kaum

${ }^{1}$ Dammer (1893) 3, 378.

Z. anorg. Chem, $1 X$. 
vorhanden, da alle diese grünen Körper dieselben Eigenschaften besitzen. Man hat ja auch bis jetzt das auf verschiedene Weise dargestellte Berliner Blau für ein und denselben Körper gehalten, obwohl sich hier Verschiedenheiten finden lassen.

LEFoRTs ${ }^{1}$ Angabe über lösliches Berliner Grün beruht jedenfalls auf einem Irrtum, da ich durch Behandeln von Eisenoxyduloxyd mit wässeriger Blausäure keine Lösung erhalten konnte, abgesehen davon, dafs nach obigen Auseinandersetzungen Berliner Grün auf diese Weise kaum entstehen kann. Man erhält nach LEFORTs Angabe wohl einen grünen Körper, allein derselbe ist nichts anderes als eine Mischung, die von gebildetem Berliner Blau und Eisenhydroxyd grün gefärbt erscheint.

\section{WILIIAMSONs Violett. ${ }^{2}$}

Das nach seinem Darsteller benannte WiLIIAMsonsche Violett hielt man auf Grund der Untersuchungen Williamsons für Ferrikaliumferrocyanid: $\mathrm{KFeFeCy}_{6}$. Ich will nun hier zunächst an der Hand der Wildiamsonschen Beschreibung dieses Körpers den letzteren in Darstellung, Eigenschaften und quantitativer Zusammensetzung näher beleuchten.

Durch Oxydation des Blausäurerückstandes (Ferrokaliumferrocyanid: $\mathrm{K}_{2} \mathrm{FeF}^{\mathrm{II}} \mathrm{eCy} \mathrm{y}_{6}$ ) entsteht ein blauer Körper, den GMeLin für Berliner Blau hielt, den Williamson als Ferrikaliumferrocyanid bezeichnet. Wirdiamsos stellte diesen Körper durch Oxydation von Blausäurerückstand mit verdünnter Salpetersäure dar und behauptete, dals auch durch andere Oxydationsmittel derselbe Körper entstehe. Allein Wiblinason hat jedenfalls keine genügenden Beobachtungen in dieser Beziehung gemacht; denn durch Oxydation mit Chlor, Brom oder Ferrisalze erbält man ganz andere Oxydationsprodukte.

Oxydiert man den Blausäurerückstand mit Chlor oder Brom, so erhält man das Violett nicht rein, sondern eine Mischung desselben mit Berliner Grün, welche eine schmutziggrüne Farbe besitzt und bei anhaltender Oxydation in Berliner Grün übergeht. Es entsteht wohl das Violett, allein dieses wird eben, bevor alles $\mathrm{K}_{2} \mathrm{FeFeCy}_{6}$ in Violett verwandelt ist, schon teilweise weiter oxydiert.

1 Juhresbericht (VIEWEG \& SOHN) (1869) 267.

${ }^{2}$ Lieb. Ann. 57, 232. 
Oxydiert man mit Ferrisalzen, so erhält man violette Körper, die weniger Kali enthalten, als der Formel $\mathrm{KFeFeCy}_{6}$ entspricht.

Nur durch Oxydation mit verdünnter Salpetersäure erhält man ein schönes Violett, wenn man genau nach Williamsons Vorschrift arbeitet. Aber ob dieses Violett der Formel $\mathrm{KFeFeCy}_{6}$ entspricht, ist sehr fraglich, wenn es auch annähernd diese quantitative $\mathrm{Zu}$ sammensetzung aufweist. Letztere beruht mehr auf Zufälligkeit und wird sehr leicht gestört, wenn zur Darstellung etwas zu viel Salpetersäure verwendet wurde, was leicht der Fall ist, da man mit überschüssiger Salpetersäure arbeiten muls. Ferner lälst sich niemals der Punkt konstatieren, bei welchem alles $\mathrm{K}_{2} \mathrm{FeFeCy}_{6}$ in Violett übergegangen ist. Entweder enthält der violette Körper noch unverändertes $\mathrm{K}_{2} \mathrm{FeFeCy}_{6}$ oder dies ist schon in geringen Mengen höher oxydiert, ohne dafs man an der Farbe des Präparates etwas sehen kann, wie bei der Oxydation mit Chlor. Auch ist die Möglichkeit vorhanden, dafs in dem Violett sowohl Blausäurerückstand als Berliner Grün zu gleicher Zeit enthalten ist, ohne dals man dafür einen Nachweis liefern könnte. WILliamson giebt zwar eine Reaktion an, mit deren Hilfe man den Punkt bestimmen kann, bei welchem die Oxydation gerade weit genug vorgeschritten ist. Er lälst eine Probe des Violetts mit Alkali zersetzen und glaubt aus den Zersetzungsprodukten einen Schlufs auf die Reinheit seines Präparates machen zu können. Entsteht dabei neben Ferrocyankali auch Ferricyankali, so ist schon zu weit oxydiert, entsteht aber Ferrohydroxyd, so mufs noch weiter oxydiert werden. Allein darin täuschte sich WrLLIAMson. Die Alkalizersetzung ist beim Violett noch komplizierter als beim Berliner Grün und man kann sich auf die Zersetzungsprodukte nicht verlassen. Wie verwickelt diese Reaktion verläuft, mag folgende kurze Betrachtung zeigen.

Wenn man das Williamsonsche Violett mit Kaliumhydroxydoder Kaliumkarbonatlösung erwärmt, so treten folgende Reaktionen ein, die sich in den meisten Fällen sehr leicht nachweisen lassen :

1. $\mathrm{KFeFeCy} y_{6}+3 \mathrm{KOH}=\mathrm{Fe}(\mathrm{OH})_{8}+\mathrm{K}_{4} \mathrm{FeCy}_{6}$,

2. $\mathrm{KFeFeCy}_{6}+\mathrm{K}_{4} \mathrm{FeCy}_{6}=\mathrm{K}_{\mathbf{8}} \mathrm{FeFeCy}_{6}+\mathrm{K}_{8} \mathrm{FeCy}_{6}$,

3. $\mathrm{K}_{2} \mathrm{FeFeCy}_{8}+\mathrm{K}_{8} \mathrm{FeCy}_{6}=\mathrm{KFeFeCy_{6 }}+\mathrm{K}_{4} \mathrm{FeCy}_{6}$,

4. $\mathrm{K}_{2} \mathrm{FeFeCy_{6 }}+2 \mathrm{KOH}=\mathrm{Fe}(\mathrm{OH})_{2}+\mathrm{K}_{4} \mathrm{FeCy}_{6}$.

Die Reaktion 2 geht in verdünnter Lösung leichter von statten als die Reaktion 3, und infolgedessen läfst sich in den Zersetzungsprodukten immer Ferrohydroxyd und Ferricyankali nachweisen. Es kann auch noch ein komplizierterer Fall eintreten, wenn in der er- 
haltenen violetten Substanz neben unverändertem Blausäurerückstand Berliner Grün enthalten ist; kurz gesagt, man findet bei näherer Untersuchung dieser etwas verwickelten Verhältnisse, dal's die Alkalizersetzung auch beim Violett wertlos ist. Die einzige Methode, Blausäurerückstand in Violett zu rerwandeln, ohne befürchten zu müssen, dafs die Oxydation zu weit oder nicht genügend weit vor sich geht, ist jedenfals die Oxydation mit Ferrisalzen. Wenn man Blausäurerückstand mit Ferrisalzlösungen erwärm̧t, so wird er in Violett verwandelt, während das zugesetzte Ferrisalz zu Ferrosalz reduziert wird. In der Wärme ist dieser Vorgang ein vollständiger, bei gewöhnlicher Temperatur geht er nur langsam und unvollständig vor sich. Giefst man die entstandene Ferrosalzlösung rom Niederschlag $a b$, wäscht denselben mit Wasser durch Dekantieren und setzt abermals Ferrisalz zu, so erkennt man daran, dafs dieses nicht mehr reduziert wird, sehr leicht, ob der Niederschlag vollständig oxydiert ist oder nicht. Tritt keine merkliche Reduktion mehr ein, so hat man wohl das Recht, anzunehmen, dal's der Blausäurerückstand vollständig oxydiert ist. Ferrisalze haben jedenfalls nicht die nötige Oxydationskraft, um das Violett weiter in Grün zu verwandeln, ich habe wenigstens eine Beobachtung in dieser Beziehung bei meinen zahlreichen Versuchen nicht machen können. Jedenfalls darf die Oxydation mit Ferrisalzen nur bei mälsiger Wärme vorgenommen werden, denn bei der Siedetemperatur wird das gebildete Violett durch Ferrisalze langsam zerstört, wobei wieder Ferrosalz entsteht.

Hat man auf diese Weise ein Violett dargestellt, so findet man, dais dasselbe bei weitem nicht dem hohen Kaligehalt entspricht, den die Formel $\mathrm{KFeFeCy}_{6}$ rerlangt. Es mögen hier einige Darstellungsweisen und die dadurch bewirkte quantitative Zusammensetzung angegeben werden. Der Kürze halber beschränke ich mich hier auf die Analysenzahlen, die auf wasserfreie Substanz umgerechnet sind, und gebe von jedem Körper nur das Mittel aus drei Analysen an.

Die in folgendem angegebenen Violette wurden nach der Darstellung mit Wasser gewaschen und im Vakuum über Schwefelsäure getrocknet.

1. Blausäurerückstand wurde nach der Angabe WiLLIAMsoss mit verdünnter Salpetersäure oxydiert.

2. Dieses Violett erhitzte ich 4 Stunden lang mit $10^{1 \%}$ Salzsäure, um daraus zu sehen, in welchem Verhältnis Eisen und Kali im Rückstand bleiben. Eine äufsere Veränderung des Violetts tritt dabei nicht ein. 
3. Blausäurerïckstand wurde mit Ferrisulfatlösung 24 Stunden bei gewöhnlicher Temperatur stehen gelassen, wobei jedenfalls eine rollständige Oxydation nicht stattfindet.

4. Blausäurerückstand wurde 10 Stunden lang mit verdünnter Ferrisulfatlösung erwärmt, wobei eine völlige Oxydation nicht erwiesen war.

5. Blausäurerückstand wurde mit Ferrisulfatlösung erwärmt, das gebildete Ferrosulfat abgegossen, mit Wasser nachgewaschen und mit Ferrisulfat weiter behandelt, bis eine merkliche Reduktion nicht mehr stattfand.

6. Das unter 5. eben beschriebene Violett wurde noch drei Tage lang mit Ferrisulfatlösung weiterbehandelt, um $z u$ konstatieren, ob der Kaligehalt des Violetts dadurch noch weiter abnimmt, was aber unter den genannten Bedingungen wenig oder gar nicht der Fall zu sein scheint.

Die Analyse dieser violetten Körper ergab folgende Resultate in Prozentzahlen ausgedrückt:

\begin{tabular}{lrrrrrrc} 
& 1. & \multicolumn{1}{c}{2.} & \multicolumn{1}{c}{3.} & \multicolumn{1}{c}{4.} & \multicolumn{1}{c}{5.} & \multicolumn{1}{c}{6.} & $\mathrm{KFeFeCy}_{6}$ \\
$\mathrm{~K}=$ & 11.68 & 8.29 & 9.77 & 7.79 & 6.95 & 6.72 & 12.70 \\
$\mathrm{Fe}=$ & 37.78 & 40.10 & 38.78 & 40.84 & 40.88 & 40.74 & 36.48 \\
$\mathrm{Cy}=$ & 50.53 & 51.72 & 51.43 & 51.37 & 52.54 & 52.36 & 50.82
\end{tabular}

Aus 1 ist ersichtlich, dafs nach Williamsons Darstellung der Kaligehalt zur Theorie auf ein Prozent nicht stimmt. Mehrere Versuche lieferten dasselbe Resultat, man erhält leichter kaliärmere Präparate. Wildiamson hat übrigens selbst etwas zu wenig Kalium gefunden und glaubte das seiner mangelhaften Analysenmethode zuschreiben zu müssen.

Aus 2 ist ersichtlich, dafs dem Violett durch Salzsäure verhältnismäfsig viel Kali, also mehr Kali als Eisen entzogen wird. Ähnlich verhält sich das Violett gegen Oxalsäurelösung. Violett 3 mit $5 \%$ Oxalsäure mehrere Stunden lang erhitzt verlor etwa ein Prozent Kali.

Vor dem Behandeln mit Oxalsäure ergab die Analyse $9.77 \% \mathrm{~K} ; 38.78 \%$ Fe; $51.43 \%$ Cy.

$\mathrm{Nach}$ dem Behandeln mit Oxalsäure ergab die Analyse $8.71 \% \mathrm{~K} ; 39.51 \% \mathrm{Fe} ; 51.94 \% \mathrm{Cy}$.

Als besonders charakteristisčh für das Violett gegenüber dem Berliner Blau bezeichnete WiLliaMson den Mangel an Kupferglanz, die grïne Farbe des in Wasser aufgeschlämmten Körpers im durchfallenden Lichte, die Unlöslichkeit in Oxalsäure und die helle Farbe. Diese Eigenschaften sind aber durchaus nicht charakteristisch für das Violett, da es auch Berliner Blau mit denselben Eigenschaften giebt.

Je nach der Darstellung erhält man Violett mit viel oder wenig Kupferglanz, ganz frei davon ist es nicht, wenn man das frisch aus Blausäurerückstand und Salpetersäure dargestellte Violett ohne durch- 
zurühren im Vakuum trocknen lälst. Dagegen zeigt es diese Erscheinung sehr auffallend, wenn man den Blausäurerückstand mit überschüssigem Ferricyankali oxydiert hat. Das aus Berliner Grün dargestellte Berliner Blan hat ebensowenig Kupferglanz, als das nach Wilimansons Vorschrift dargestellte Violett.

Die grüne Farbe, welche das Williamsonsche Violett im durchfallenden Lichte zeigt, findet sich auch bei dem oben unter 4 und 6 beschriebenen Berliner Blau und bei anderen.

Ebenso unlöslich in Oxalsäure als das WiLuramsonsche Violett sind die aus Berliner Grün dargestellten Berliner Blau.

Das oben unter 4 beschriebene Berliner Blau ist unter Umständen heller als das Wrimiamsonsche Violett. Dieses Blau widerlegt auch die Ansicht Wiluraysons, dafs die kalireichsten Berliner Blau am hellsten, die kalifreien am dunkelsten seien, denn es ist trotz seiner sehr schönen hellen Farbe vollkommen kalifrei.

Aus den kurzen Angaben über Eigenschaften, Zusammensetzung und Darstellung, die ich durch eine grölsere Anzahl von Versuchen und Analysen noch erweitern könnte, geht schon auf das bestimmteste hervor, dals das WiLliamsonsche Violett ein Körper von sehr fraglicher Individualität ist. $\mathrm{Ob}$ die Verbindung $\mathrm{KFeFeCy}_{6} \mathrm{mehr}$ oder weniger im WiLLIAMsonschen Violett enthalten ist, oder ob dieser Körper überhaupt nicht existiert, also das Violett hauptsächlich aus kalihaltigem Berliner Blau besteht, läfst sich nicht feststellen. Die Behauptung, dafs das Wiulanmsonsche Violett Ferrikaliumferrocyanid sei, gründet sich nur auf die zufällige Zusammensetzung des durch Salpetersäure oxydierten Blausäurerückstandes und auf die theoretische Erwägung, dafs aus Ferrokaliumferrocyanid ein Ferrikaliumferrocyanid entstehen mülste, abgesehen davon, dals man auch dann nicht entscheiden könnte, ob das Violett Ferrikaliumferrocyanid oder Ferrokaliumferricyanid wäre.

\section{Nachtrag $z \mathfrak{u}$ den krystallisierten Kupferferrocyaniden.}

Aufser den schon beschriebenen Kupferferrocyaniden habe ich auch das Lithiumcupro- und Lithiumcupriferrocyanid dargestellt. Das Lithiumcuproferrocyanid erhält man durch Kochen von Kupfercyanür mit Lithiumferrocyanid in einer Wasserstoffatmosphäre, auf Zusatz von wenig schwefligsaurem Lithium, als farblose sechsseitige Prismen oder als Würfel. Das Lithiumcupriferrocyanid erhält man durch Kochen von alkalifreiem Ferrocyankupfer mit Lithiumferrocyanid als 
sehr kleine quadratische rote Blättchen, zuweilen auch als kurze braunrote Nadeln. Es glückt jedoch nicht immer, die Krystalle des Lithiumcupriferrocyanids so grols zu erhalten, dals man sie deutlich erkennen kann.

Bei Versuchen, die den Kupferferrocyaniden analogen Ferricyanide darzustellen, erhielt ich das Kaliumcupriferrocyanid. Kocht man Kupfercyanür mit uberschüssiger Ferricyankalilösung, so entsteht ein brauner krystallinischer Niederschlag, der hauptsächlich aus $\mathrm{K}_{2} \mathrm{CuFeCy}_{6}$ besteht, und eine Lösung, aus der sich beim Erkalten braunrote quadratische Prismen ausscheiden, dieselbe Form wie beim $\mathrm{Na}_{2} \mathrm{CuFeCy}_{6}$ beschrieben, nur nicht so scharf ausgebildet. Der Vorgang ist jedenfalls folgender:

$$
\mathrm{CuCy}+2 \mathrm{~K}_{8} \mathrm{FeCy}_{6}=\mathrm{K}_{8} \mathrm{CuFeCy}_{6}+\mathrm{K}_{4} \mathrm{FeCy}_{6}+\mathrm{CN} \text {. }
$$

Das Kupfercyanür wird durch das Ferricyankali oxydiert und letzteres zu Ferrocyankali reduziert. Das im Überschufs vorhandene Ferricyankali verhindert die reduzierende Wirkung des gebildeten Ferrocyankaliums.

Durch Kochen von Kupfercyanür mit Natriumferricyanid erhielt ich ebenfalls Natriumcupriferrocyanid.

Durch Kochen von Ferrocyankupfer mit Ferricyankali konnte ich keine krystallisierte Verbindung erhalten.

Bei der Redaktion eingegangen am 25. März 1895. 